\title{
Vulnerability Analysis of Power Grids Using Modified Centrality Measures
}

\author{
Francisco Gutierrez, E. Barocio, F. Uribe, and P. Zuniga \\ Graduate Program in Electrical Engineering at CUCEI, Department of the State University of Guadalajara, \\ Guadalajara, Jalisco, Mexico
}

Correspondence should be addressed to E. Barocio; emilio.barocio@cucei.udg.mx

Received 9 December 2012; Revised 22 February 2013; Accepted 25 February 2013

Academic Editor: Ricardo Femat

Copyright (C) 2013 Francisco Gutierrez et al. This is an open access article distributed under the Creative Commons Attribution License, which permits unrestricted use, distribution, and reproduction in any medium, provided the original work is properly cited.

\begin{abstract}
The aim of this paper is to propose modified centrality measures as a tool to identify critical nodes before a vulnerability analysis is performed in an electrical power grid. Pair dependency centrality is weighted using the grid active power flow, and this becomes the basis to define closeness and betweenness of its nodes, and hence to identify the most critical ones. To support the idea of using modified centralities, four power grids are tested to be either exponential or scale-free. To evaluate the proposal, information obtained via modified centrality measures is used to calculate global efficiency of the power grids.
\end{abstract}

\section{Introduction}

Today more than ever, electrical energy has become a key commodity to any growing society. Efficient and reliable supply of this energy is the main concern of the electrical companies. However, the increasing demand, the incorporation of intermittent generation, the deregulation of the power system services, and more recently the climate impacts are pushing the power system close to unstable operating conditions. In this regard, control and measurement systems as well as back up policies are being developed towards ensuring an efficient and reliable service. Following the trend, vulnerability analysis tools aim to an effective identification of those nodes critical to the stability of power systems, which is useful to define device placement and can draw guidelines for the expansion of an electric network. Contingency and transient stability analysis techniques are also used for dynamic security studies; however, as power systems grow and become more complex $[1,2]$, the number of contingencies increases significantly, with the consequent raise in computation time [3]. This has motivated research towards the development of new analysis techniques.
Some of the techniques proposed in recent years use concepts from network theory to study several properties of power grids. An index based on the connection pattern to measure how close a power grid is to be a small world network, and hence evaluating its vulnerability is proposed in [4]; using that same connection pattern [5] shows which small world characteristics are hold by power grids. The authors in [6-8] propose global efficiency as a measure to define vulnerable nodes in a power grid and to propose improvements. Betweenness as a vulnerability index is used in [9], where power grids are classified as scale-free. These works show that very few important characteristics of power grids can be spotted using concepts originally developed for the complex networks framework, hence more specific power grid focused metrics must be developed in order to represent key characteristics and complement or improve state of the art techniques dedicated to power grid vulnerability analysis. To overcome this deficiency, [10] proposes an index based on the ratio of loads disconnected after a contingency to find vulnerable nodes while [11] uses power transfer distribution factors and transmission line limits to define new metrics that evaluate power grid vulnerability. Impedance of transmission 


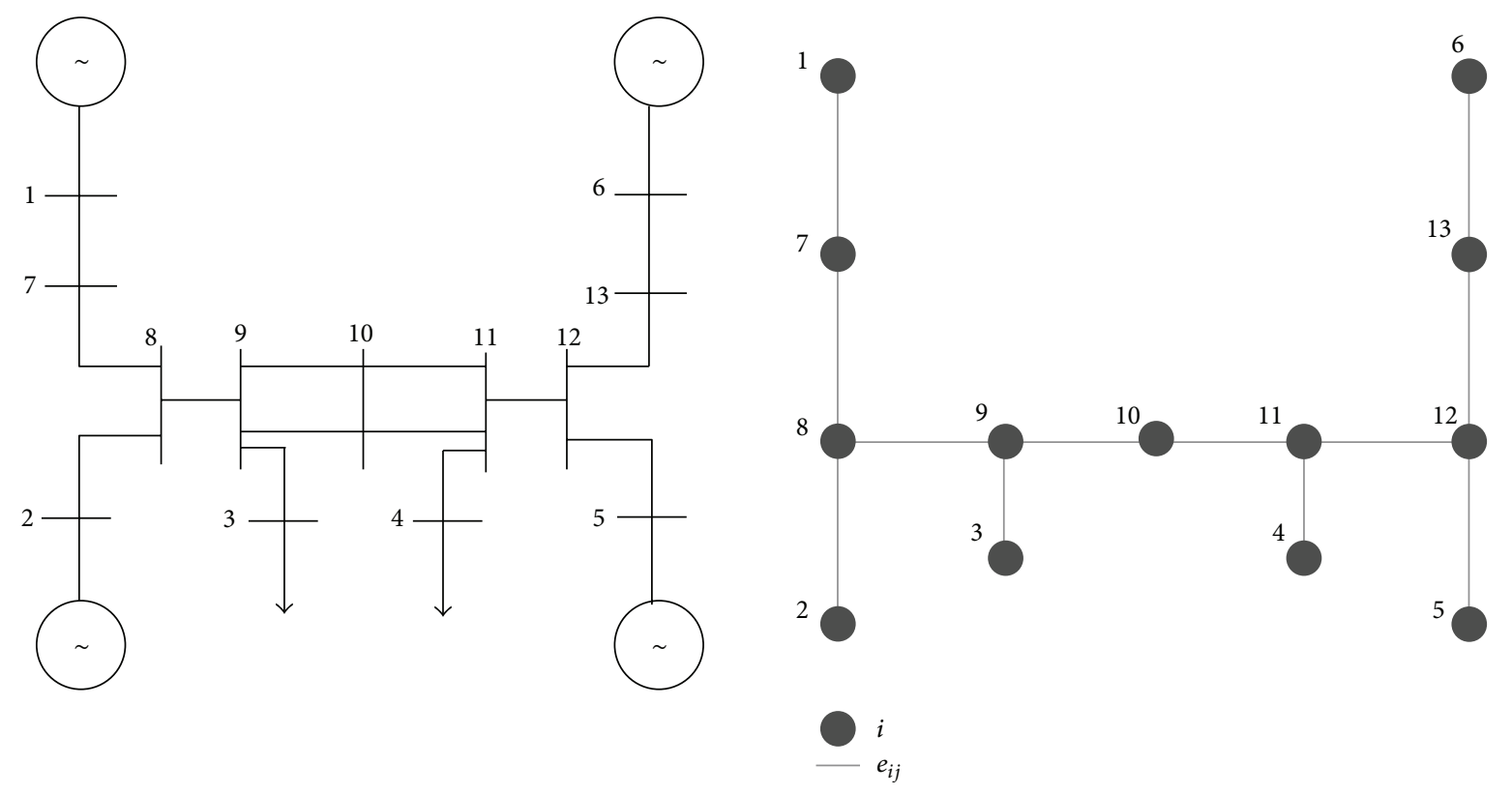

FIGURE 1: A power system and its correspondent graph.

lines and a weighted Laplacian are employed as a base to define betweenness in [12] and closeness in [13], respectively; in these cases, centrality measures are redefined using different "electrical distances."

In this work, modified centrality measures are proposed in the complex networks framework as an extension of the results presented in $[14,15]$, taking into consideration operative restrictions in the voltage and power flow limits. The active power flow is selected in this analysis due to represent most of its behavior in steady state. In this way, proper information is used to detect critical nodes in a single simulation run. It is important to mention that reactive power flow information can also be considered in this modified centrality measures; however, due to space limitation their results were not included in this paper.

The outline of the paper is as follows: first the derivation of the proposed modified centrality measures is presented, then the calculation of these measures is applied to four test power grids given, and the results are given a physical interpretation. Finally, the proposed metrics are compared with other definitions of the same centralities found in the literature, in order to determine its strengths and weaknesses, and the power grid vulnerability is evaluated using the information obtained.

\section{A Graph Representation of Power Grids}

Graphs are useful as a tool to obtain a representation of the interconnections between the components of a network. A graph $\mathscr{G}$ consists of a set of vertices (also called sites, actors, points, or nodes) representing the elements of the network, a set of edges (also called bonds, ties, lines, or branches) that describe the nature of network interconnections, and a set of edge weights that specify their intensity.
As shown in Figure 1, a power grid can easily be represented by a graph considering its $N$ nodes as the graph vertices, and its weighted interconnections as the impedances of the $l$ transmission lines connecting them. Hereafter, graph vertices are called nodes, and the corresponding weighted interconnections are given by the absolute value of transmission line impedance. Also, the graph representation of a power grid is an undirected (interaction between two adjacent nodes is reciprocal (two nodes are said to be adjacent if there exists $\left.e_{i j}\right)$ ) and connected (there is a path between any pair of nodes) graph of order (the number of nodes in $\mathscr{G}$ is called the order of $\mathscr{G}$ ) $N$ and size (the number of lines in $\mathscr{G}$ is called the size of $\mathscr{G}) l$ as follows:

$$
\mathscr{G}=\{\mathcal{N}, E, Z\}
$$

where the set $\mathcal{N}=\left\{i \mid i \in \mathscr{N}^{N}\right\}$ includes all nodes of the power grid, the set $E=\left\{e_{i j} \mid e_{i j} \in E^{l} \bigcap i, j \in \mathcal{N}^{N}\right\}$ describes the transmission lines connecting them, and the set of weights $Z=\left\{\left|z_{i j}\right||| z_{i j} \mid \in Z^{l} \cap i, j \in \mathcal{N}^{N}\right\}$ represents the electrical distance linking each pair of nodes.

\section{Modified Centrality Measures}

Centrality measures can generally be defined as functions that assign a real value to each node in a network in order to identify its participation in a certain process. In this section, modified centrality measures are proposed based on the original ones defined in the complex networks framework, then its adaptation to power systems is presented.

For the graph representation used in this work only active power flow between nodes and a steady-state operation are considered. It is important to notice that (as proposed in [14]) the type of node, that is, generation, transmission, or load, cannot be distinguished from one another. 
3.1. Power Flow Formulation. Let vector $\mathbf{S}$ denote the injection of power into an electric grid, defined as a function of voltage and current in each node as follows:

$$
\mathbf{S}=\mathbf{V}_{\text {diag }} \mathbf{I}^{*}
$$

where $\mathbf{V}_{\text {diag }}=\operatorname{diag}\left(V_{i}\right)$ and $S_{i}, I_{i}, V_{i} \in \mathbb{C}^{N}$ are the power, current, and voltage at node $i$, respectively. Then, the vector of injected currents is

$$
\mathbf{I}=\mathbf{Y V},
$$

where $\mathbf{Y}$ is the admittance matrix of the power grid [16] and each element is defined as $Y_{i j}=1 / Z_{i j}$. Substituting current from (3) in (2), one has

$$
\mathbf{S}=\mathbf{V}_{\text {diag }}\left(\mathbf{Y}^{*} \mathbf{V}^{*}\right) .
$$

Expanding (4), a nonlinear complex algebraic expression is obtained for each node as follows:

$$
S_{i}=V_{i} \sum_{j=1}^{N} V_{j} e^{-\theta_{i j}} Y_{i j} .
$$

Expanding and rearranging (5) taking into account that $e^{-j \theta_{i j}}=\cos \theta_{i j}+j \sin \theta_{i j}, S_{i}=P_{i}+j Q_{i}$, and $Y_{i j}=G_{i j}+j B_{i j}$ lead to (6) that represent active and reactive powers in each node, respectively, as a function of their voltages and phase angles and the connectivity between them as follows:

$$
\begin{aligned}
& P_{i}=V_{i} \sum_{j=1}^{N} V_{j}\left(G_{i j} \cos \theta_{i j}+B_{i j} \sin \theta_{i j}\right), \\
& Q_{i}=V_{i} \sum_{j=1}^{N} V_{j}\left(G_{i j} \sin \theta_{i j}-B_{i j} \cos \theta_{i j}\right) .
\end{aligned}
$$

Since only active power flow is considered in this work, the steady-state power transfer in any transmission line is given by

$$
P_{i j}=V_{i} V_{j}\left(G_{i j} \cos \theta_{i j}+B_{i j} \sin \theta_{i j}\right)+V_{i}^{2} G_{i j}
$$

Subject to: 1.05 p.u. $\geq V_{i}, \quad V_{i} \geq 1.05$ p.u.

$$
P_{i j} \leq P_{i j \max } \text {. }
$$

Figure 2 shows the transmission model line used. Also, if the active power is transmitted from $i$ to $j$ and $r_{i j} \neq 0$, then $\left|P_{i j}\right|>$ $\left|P_{j i}\right|$.

3.2. Pair Dependency. Pair dependency was first introduced by Freeman in [15] to find a set of nodes whose position in the network allows them to "facilitate, inhibit, or distort" the exchange of information. Following this idea, the derivation of a modified pair dependency centrality is presented in this section.

A recursive implementation of Dijkstra's algorithm [17] is employed in this work to find the shortest path between a pair of nodes $(n, m)$ in the power grid in order to calculate

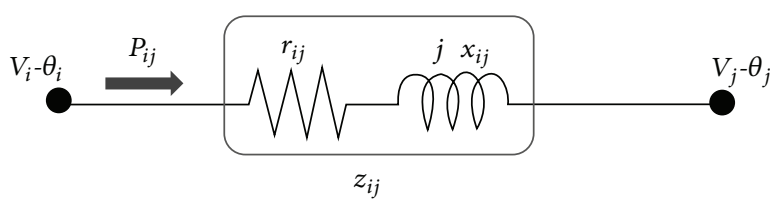

FIgURE 2: Transmission line scheme.

its maximum active power (The shortest path between two nodes is the succession of lines connecting them, in such a way that they sum to the least possible weight and that no node or line appears two times), given by

$$
P_{n m \max }=\max \left\{\left|P_{i j}\right| \mid i, j \in g_{n m} \subseteq V\right\},
$$

where $g_{n m}$ is the set of nodes included in the shortest path connecting $n$ and $m$.

At each node $k \neq i, j \in g_{n m}$, an expression for the maximum active power injection of the shortest path is calculated as follows:

$$
P_{n m}(k)=\max \left\{\left(\left|P_{i k}\right|,\left|P_{k j}\right|\right) \mid i, j, k \in g_{n m} \subseteq V\right\} .
$$

The ratio of $P_{n m}(k)$ to $P_{n m \max }$ is the degree in which buses $n$ and $m$ need bus $k$ to transmit active power along the shortest electrical path as follows:

$$
r_{n m}(k)=\frac{P_{n m}(k)}{P_{n m \max }} .
$$

If all shortest paths starting at $n$ are calculated, pair dependency is defined as

$$
d_{m k}=\sum_{m=1}^{N} \frac{P_{n m}(k)}{P_{n m \max }} .
$$

This represents the dependency of node $m$ to node $k$ for transmission of active power through the shortest path.

All pair dependency combinations are stored in a matrix as follows:

$$
D=\left[\begin{array}{cccccc}
d_{11} & d_{12} & \ldots & d_{1 k} & \ldots & d_{1 N} \\
d_{21} & d_{22} & \ldots & d_{2 k} & \ldots & d_{2 N} \\
\vdots & \vdots & \ddots & \vdots & \ddots & \vdots \\
d_{m 1} & d_{m 2} & \ldots & d_{m k} & \ldots & d_{m N} \\
\vdots & \vdots & \ddots & \vdots & \ddots & \vdots \\
d_{N 1} & d_{N 2} & \ldots & d_{N k} & \ldots & d_{N N}
\end{array}\right]
$$

It can be noticed that $D$ is an asymmetrical partially full matrix with zero main diagonal and is the basis to define closeness and betweenness centralities.

3.3. Closeness. In a topological framework, closeness centrality of a node is defined as the sum of all its shortest paths and can be used to quantify how rapidly the information injected in each node spreads in the network [18] by measuring how "far" a node is from the rest. Analogously to [15], closeness of a node $m$ is given by

$$
C_{c}(m)=\sum_{n=1}^{N} d_{m k}=\sum_{k=1}^{N} \sum_{n=1}^{N} \frac{P_{n m}(k)}{P_{n m \max }} .
$$




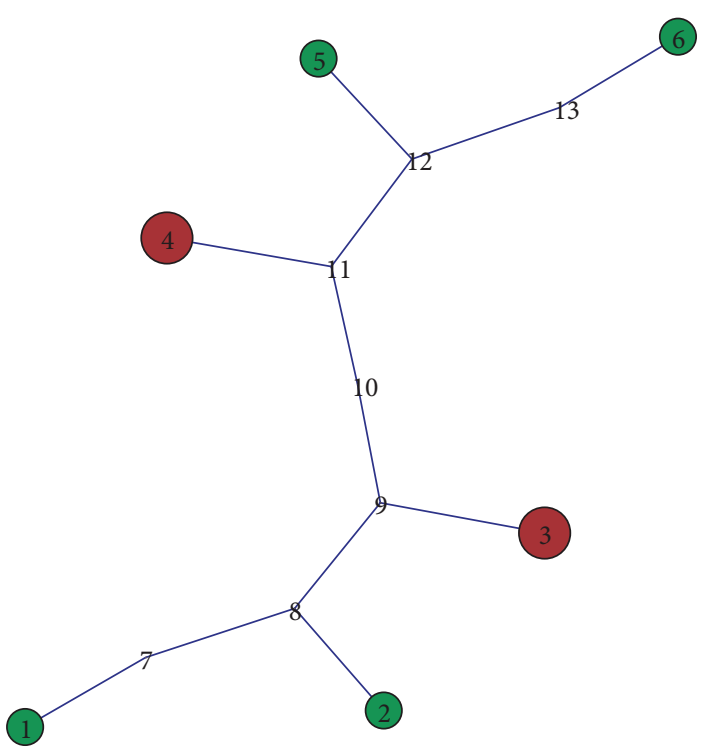

(a)

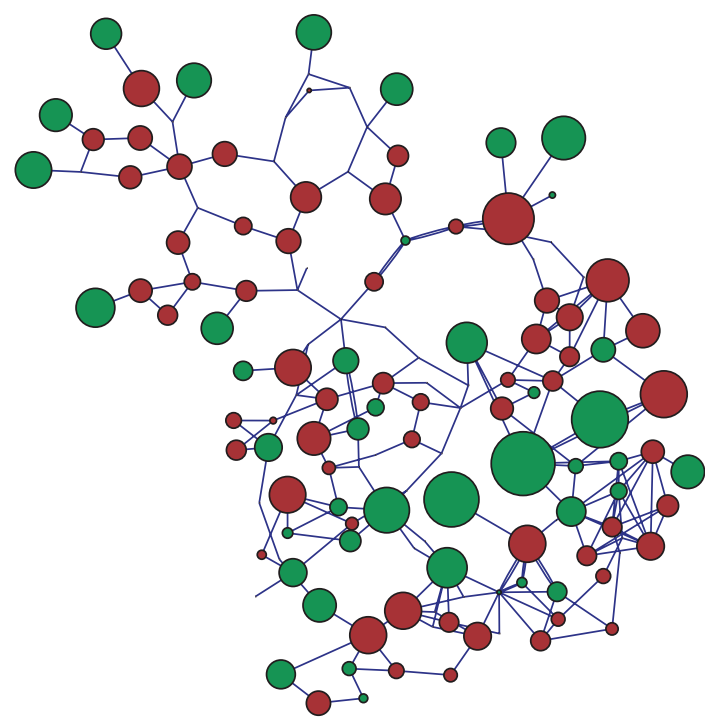

(c)

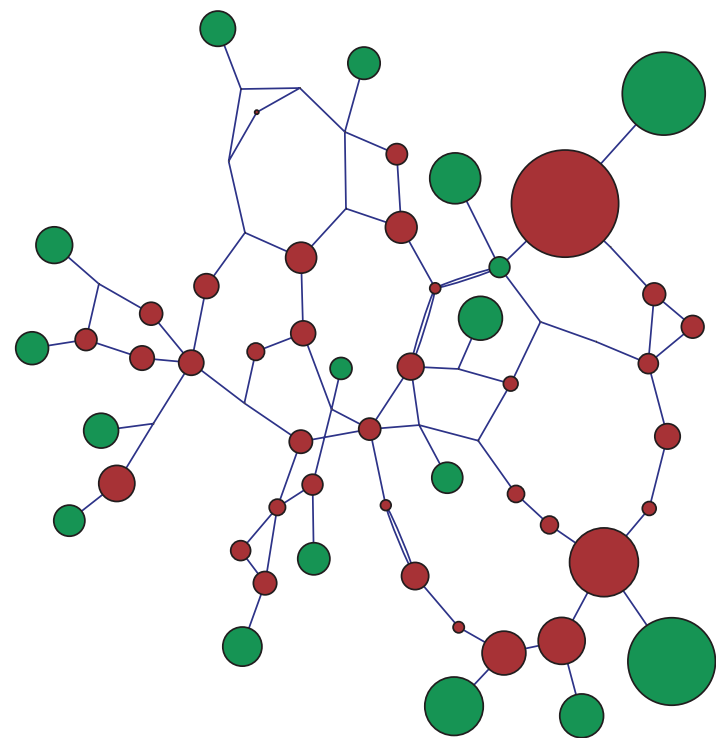

(b)

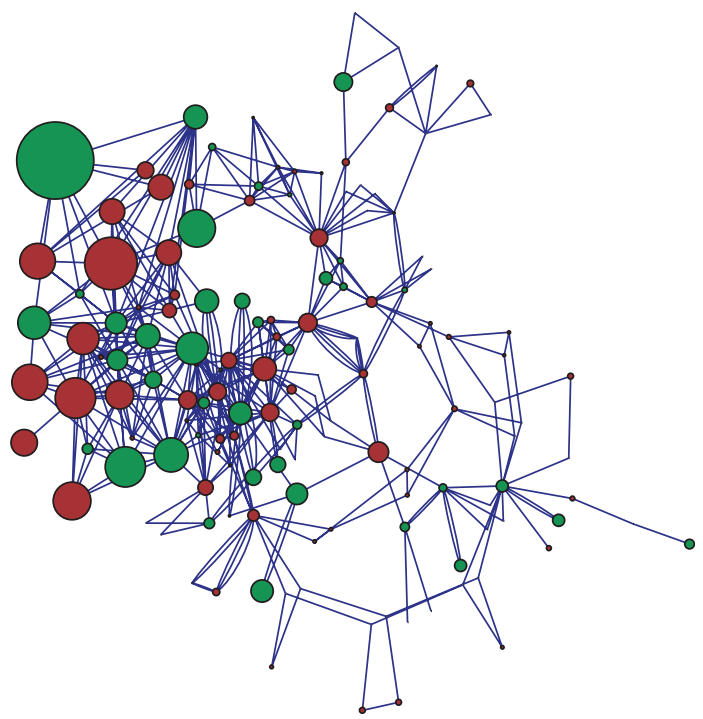

(d)

Figure 3: Test power grid multigraphs (a) $\mathscr{G}_{4}$, (b) $\mathscr{G}_{16}$, (c) $\mathscr{G}_{48}$, and (d) $\mathscr{G}_{50}$. Nodes are scaled as a function of the MW injected (generators, green dots) or withdrawn (loads, red dots). Since transmission nodes inject zero active power, they are scaled to zero but can be recognized as junctions or direction changes of lines, for example, nodes 11 and 7, respectively, in (a).

According to [14], closeness of node $m$ is given by the sum of the elements in the $m$ th row of $D$; therefore, nodes with smaller $C_{C}(m)$ are considered as closer to each other.

3.4. Betweenness. In complex network studies, betweenness measures the ratio and total number of shortest paths in a graph [19], and as a result, nodes with high values of the metric can be designated to control or regulate information flowing within a network [15]. However, when applied to power grids, the measure indicates how frequently a certain node $k$ is involved in the transmission of active power and is defined by

$$
C_{c}(k)=\sum_{m=1}^{N} d_{m k}=\sum_{m=1}^{N} \sum_{n=1}^{N} \frac{P_{n m}(k)}{P_{n m \max }}
$$

As stated in [15], the index $C_{c}(k)$ of a node is the sum of the elements in the $k$ th column of $D$.

As can be observed, pair dependency and betweenness centralities have a similar structure; however, the difference is that the former relates a node with a given pair of nodes (specific interaction), while the latter represents the dependency between a given node and all remaining ones (global interaction). 
TABLE 1: Test power grids components, number of nodes $N$, and transmission lines $l$ comprising each grid are presented.

\begin{tabular}{lcc}
\hline Power grid & Range $(N)$ & Size $(l)$ \\
\hline $\mathscr{G}_{4}$ & 13 & 12 \\
$\mathscr{G}_{16}$ & 68 & 86 \\
$\mathscr{G}_{48}$ & 140 & 233 \\
$\mathscr{G}_{50}$ & 145 & 453 \\
\hline
\end{tabular}

\section{Description of the Test Power Grids and Its Classification in the Complex Networks Framework}

The test power grids are presented from a topological perspective, firstly, described regarding the connection pattern and the number of nodes and transmission lines, secondly, classified either as exponential or scale-free using the cumulative distribution function (CDF) of its node degree.

4.1. Test Power Grids Description. Figure 3 shows the graphs corresponding to the test power grids, and Table 1 lists the number of nodes, transmission lines, and generators comprising them. A modification has been made to the power grid graphs in order to visually identify generation (clear dots), transmission (junctions or direction changes of lines), and load (dark dots) nodes.

As can be seen in Figure 3(a), the four generators power grid $\left(\mathscr{G}_{4}\right)$ is symmetrical, radial and divided into two areas connected through a transmission corridor formed by the junctions at the center of the figure, and since it is symmetric, similar centralities are expected in certain nodes. As shown in Figure 3(b), $\mathscr{G}_{16}$ has both meshed and radial zones. Figures 3(c) and 3(d) show the graphs of the test power grids $\mathscr{G}_{48}$ and $\mathscr{G}_{50}$, respectively. These are the biggest power grids analyzed and are basically meshed. They have a similar number of nodes and generators but very different number of transmission lines.

4.2. Test Power Grids Classification. Classifying networks into general groups is a tendency in complex networks since it allows finding similarities between different kinds of networks, [20] and reveals important characteristics related to their structure. In this section, the test power systems are classified either as scale-free or exponential type, in order to show the necessity of considering information related to power grids nature.

Although there are different ways to classify networks (clustering coefficient, average length path, etc.) that are based only in the subsets $\mathcal{N}$ and $E$ of $\mathscr{G}$, the node degree (d) cumulative distribution is used in this work as a basis to categorize each network (the number of lines incident with a node is called degree in Figure 3(a), node 9 has degree three, while node 4 has degree one). The CDF curves for each test power grids are shown in Figure 4.

If the CDF fits a power law function; that is, it can be described by $P(X \geq d)=f(d) \sim d^{\zeta}$, the network is said to be scale-free; according to [5], in most networks arising
TABLE 2: Fittings of the CDF curves of test power grids using MATLAB's curve fitting tool.

\begin{tabular}{ccccccc}
\hline & \multicolumn{3}{c}{ Exponential $\left(P(X \leq d)=x e^{-d \xi}\right)$} & \multicolumn{3}{c}{ Scale-free $\left(P(X \geq d)=z d^{\zeta}\right)$} \\
& $x$ & $\xi$ & $R^{2}$ & $z$ & $\zeta$ & $R^{2}$ \\
\hline $\mathscr{G}_{4}$ & 2.048 & -0.7011 & 0.96 & 1.025 & -1.2490 & 0.91 \\
$\mathscr{G}_{16}$ & 1.774 & -0.5051 & 0.93 & 1.081 & 1.0390 & 0.82 \\
$\mathscr{G}_{48}$ & 1.620 & -0.3832 & 0.96 & 1.138 & -0.9649 & 0.81 \\
$\mathscr{G}_{50}$ & 1.251 & -0.1908 & 0.99 & 1.189 & -0.7395 & 0.86 \\
\hline
\end{tabular}

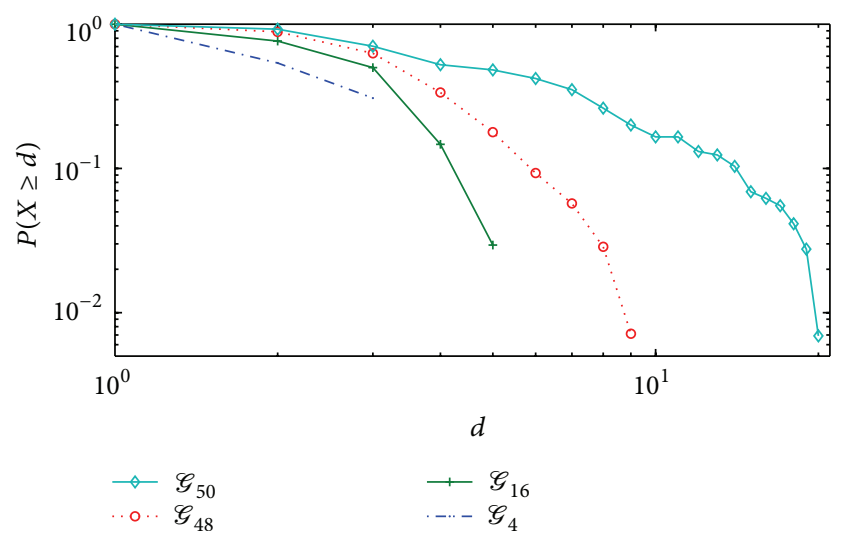

Figure 4: CDF of test power grids.

from real life, coefficient $\zeta$ is bounded $2 \leq \zeta \leq 3$. In such a network, a few nodes called hubs have a major number of connections [21], while the rest only has a few. Hence, it is said to be vulnerable to targeted attacks but resistant to random ones $[22,23]$ because the network structure is damaged to a bigger extent if hubs fail (many nodes will be disconnected, impairing an adequate information exchange).

On the other hand, if the CDF curve fits an exponential function $P(X \leq d)=f(d) \sim e^{-d \xi}$, the network is considered exponential. In this case, most nodes in the network have a similar degree. Then, when high degree nodes are disconnected, its adjacent nodes usually remain connected to the network through some other line. Hence this kind of network is said to be less vulnerable to both random and targeted attacks $[22,23]$.

Table 2 shows the results of both fittings, where it is clear that the test power grids have a better fit in the exponential classification (although scale-free fitting has a fairly high correlation coefficient, $R^{2}$ ). The difference between both fittings can be spotted in Figure 5, where the exponential curve (Exp) fits the CDF of test power grid $\mathscr{G}_{50}$ better than the scale-free curve (SF).

Results shown in Table 2 indicate that the topology of the test power grids is barely vulnerable to either random or targeted attacks. However, a single failure in a power grid can initiate a series of events leading to a major damage, for example, what is reported in [24]. Therefore, weak points cannot be detected using only the subsets of $\mathscr{G}$ related to pattern connection, and other physical characteristics of power systems must be considered when using a complex network framework. 


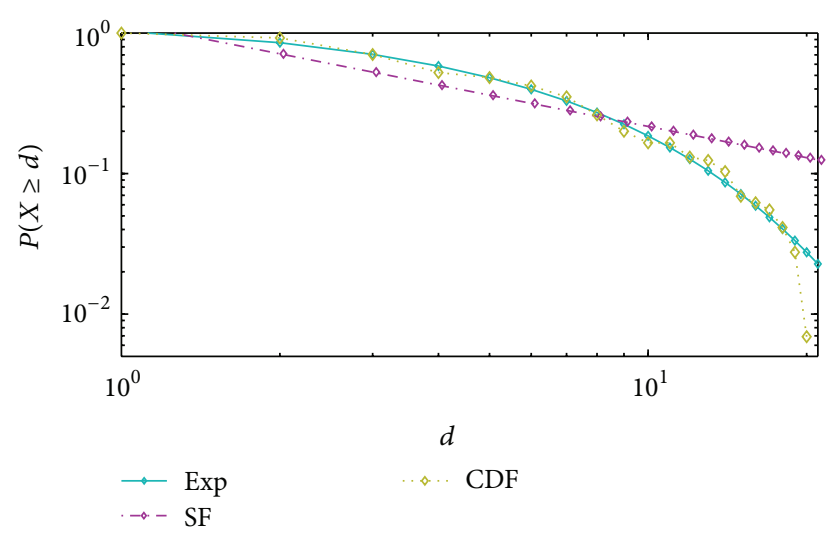

Figure 5: Exponential (Exp) and scale-free (SF) fits for the CDF of $\mathscr{G}_{50}$.

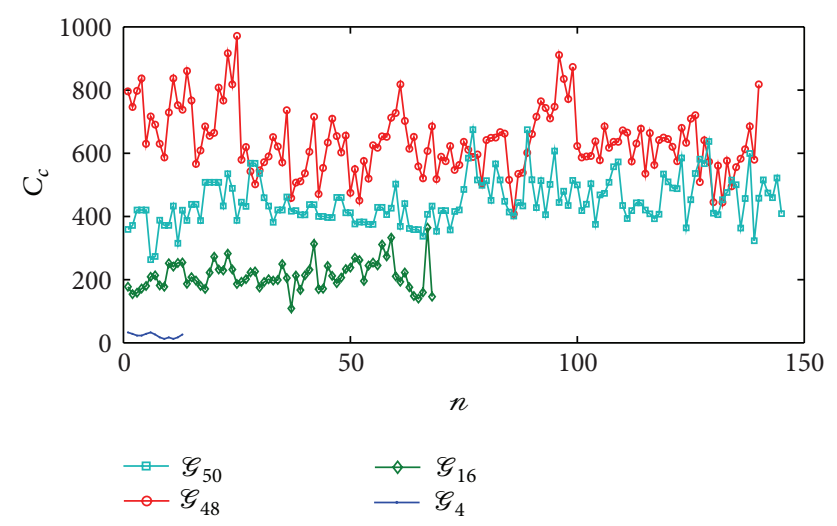

FIGURE 6: Closeness of the test power grids.

\section{Description and Comparison of Modified Centrality Measures in Test Power Grids}

In this section, results found using modified centrality measures in the test power grids are presented. First, discussions of their interpretation are drawn, and the indices obtained are normalized and compared with the widely used betweenness and closeness proposed originally in $[25,26]$ and joined by Freeman in [15], and also against two recent proposals defining these same concepts as a function of the electrical distances of power grids $[12,13]$.

5.1. Closeness of the Test Power Grids. Closeness indices obtained in all four test power grids, shown in Figure 6, have particular characteristics to notice. The first is that in a certain power grid all nodes have a similar index; however, it tends to augment, as the power grid range increases. This particularity is inherited from the exponential nature of electric networks as a consequence of the homogeneousness presented by the node degree, and deeper insight of this characteristic can be found in [27].

The second observation is that as a power grid becomes meshed, its nodes get closer, and this is apparent when comparing closeness indices in power grids $\mathscr{G}_{50}$ and $\mathscr{G}_{48}$. This particularity connects directly with the relationship between $l$ and $N$, where a bigger ratio $l / N$ means that there are more possibilities of going from one node to another, and hence the nodes in the power grid are closer [28]. Although these observations may seem trivial, they enclose the effect of topology over the closeness index that is influenced mainly by power grids topology, rather than by the power flowing through it.

Results obtained with the modified index are compared with the original definition of closeness and a redefinition based on the work presented in [13]. The former uses only the node-line information of the graph (sets $\mathscr{N}$ and $E$ ), while the latter uses the effective resistance $\left(R_{e}(i, j)\right)$ between nodes to define closeness as $S_{2}(i)=1 / \sum_{j} R_{e}(i, j)$.

As it has been pointed out, modified closeness retains the connection pattern of the grid, and because of this, results from both modified and original indexes (presented in Figure 7) are similar for all test grids. On the other hand, closeness centrality based on effective resistance has a similar behavior in small power grids but differs in large ones. In $\mathscr{G}_{50}$, for example, nodes $34,35,36$, and 99 are designated as the closest; however, they are far from the rest of the grid (detail in Figure 3(d)). In this case, all these nodes are connected through short lines with impedances much lower than the average in the network $z_{36-99} \ll \bar{z}$, which produces a poorly conditioned inverse matrix.

5.2. Betweenness of the Test Power Grids. Graphs corresponding to the test power grids, scaled using the normalized betweenness index $\left(C_{B}\right)$ of each node, are shown in Figure 8.

It can be noticed in the upper right side of Figure $8(\mathrm{~b})$ that the beginning of a radial portion of the power grid leads to a big dependence of the node connecting it. In Figure 8(c), an important transmission zone can be detected as a bundle of nodes with similar betweenness. Finally, Figure 8(d) shows how a node takes great importance by laying in a big number of shortest paths.

The indices $\left(C_{B}\right)$ calculated are compared with results using its original definition and with the modification proposed in [12], which uses the diagonal values $z_{i i}$ of the $Z_{\mathrm{Bus}}$ matrix as the node betweenness. Figure 9 shows the cumulative distribution function of the normalized node index obtained with each definition. In the first case, impedance based centralities have a different distribution compared with the other two indices, which means that the first considers all nodes having a similar betweenness. In this case, the modified index results in a close to zero magnitude for node 10, while the original and impedance based definitions have a value close to that of nodes 9 and 11 . Since power flow through node 10 is low, a failure in this node would not interrupt the service to the loads, making the grid resistant to node failures.

When observing the rest of the CDFs, a pattern similar to that of exponential networks is visible, and although there are some nodes with high betweenness, these power grids can be considered robust in general. However, nodes with higher values of this centrality should be considered critical to active power transmission and are candidates for control device allocation to effectively modify active power transfer. 


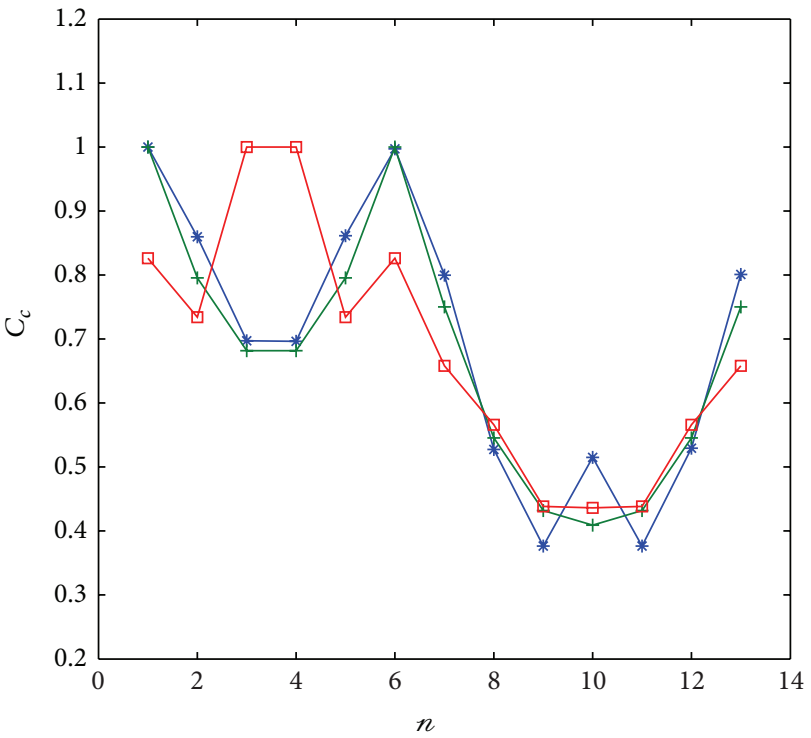

(a)

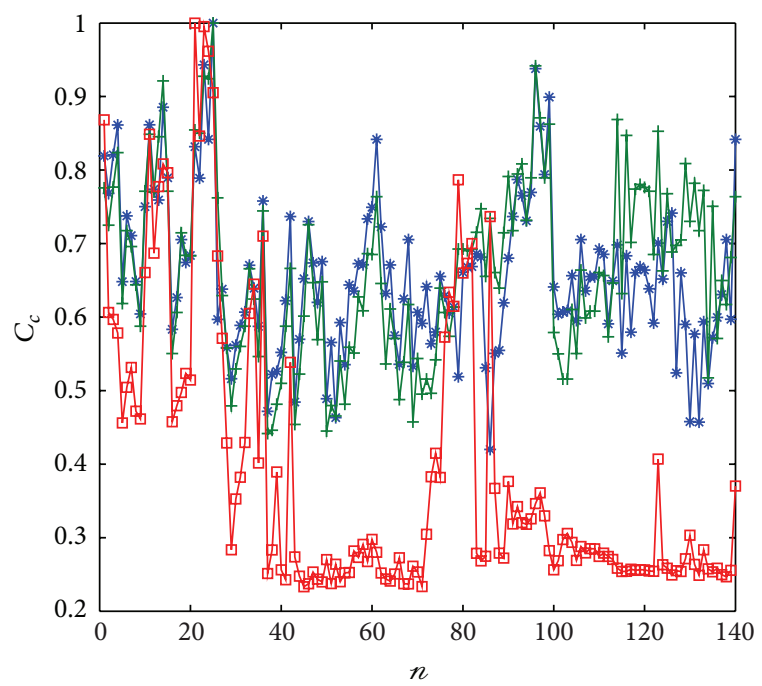

(c)

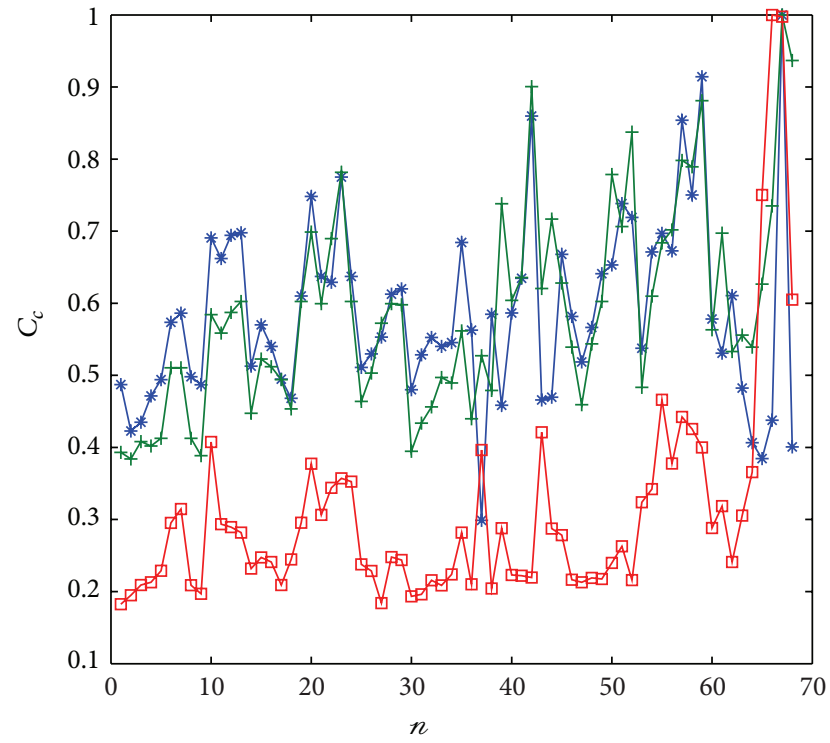

(b)

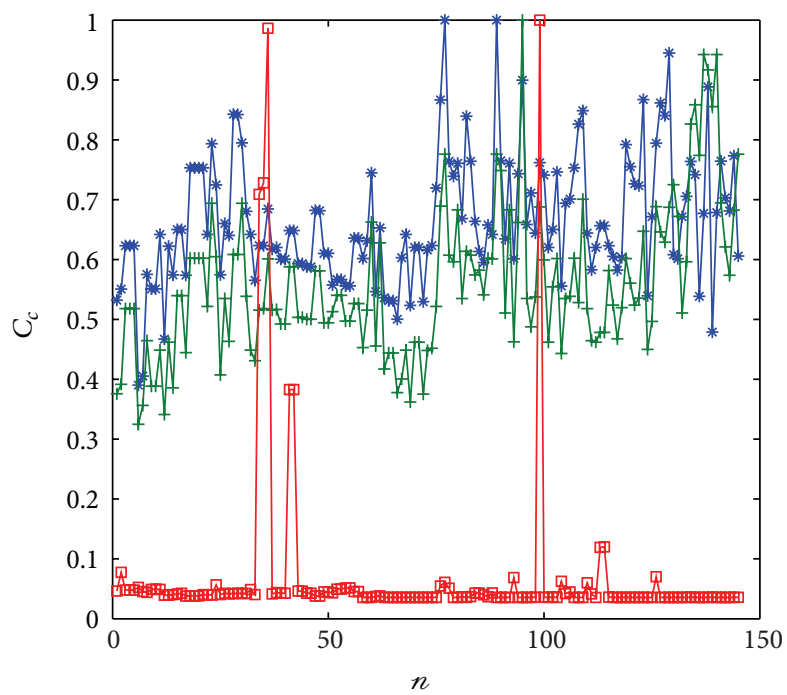

(d)

Figure 7: Closeness indices of test power grids (a) $\mathscr{G}_{4}$, (b) $\mathscr{G}_{16}$, (c) $\mathscr{G}_{48}$, and (d) $\mathscr{G}_{50}$ obtained using three different definitions: the original definition -+- , the proposed modification $-*-$, and the centrality based solely on $R_{i j}-\square-$.

After analyzing the results obtained, the test power grids seem to be robust to some extent, but, as it will be tested in the next section, nodes detected as critical via modified centrality measures are still expected to produce major damages than one chosen randomly.

\section{Vulnerability Analysis via Global Efficiency}

In order to evaluate if nodes identified using modified centrality measures have a mayor impact in the performance of the electric network, vulnerability of the test power grids is analyzed via their global efficiency. This metric was first introduced in [29] to measure the performance of a network under topological changes, and it only takes into account its graph. Here it is used as a first insight to evaluate power system vulnerability. The metric is defined as [29]

$$
E(\mathscr{G})=\frac{1}{N(N-1)} \sum_{n \neq m}^{N} \frac{1}{d_{n m}}=\frac{1}{N(N-1)} \sum_{n \neq m}^{N} e_{n m},
$$

where $d_{n m}$ is the length of the shortest path connecting nodes $n$ and $m$, that is, the sum of all $z_{i j}$ included in $g_{n m}$, and $e_{n m}$ is the efficiency between nodes $n$ and $m$.

As can be seen in (15), global efficiency of a network is given by the average sum of the inverse of all shortest paths between its nodes; therefore, a network is more efficient if its shortest paths get smaller (or $e_{n m}$ gets bigger). However, a value of global efficiency is representative only with respect 


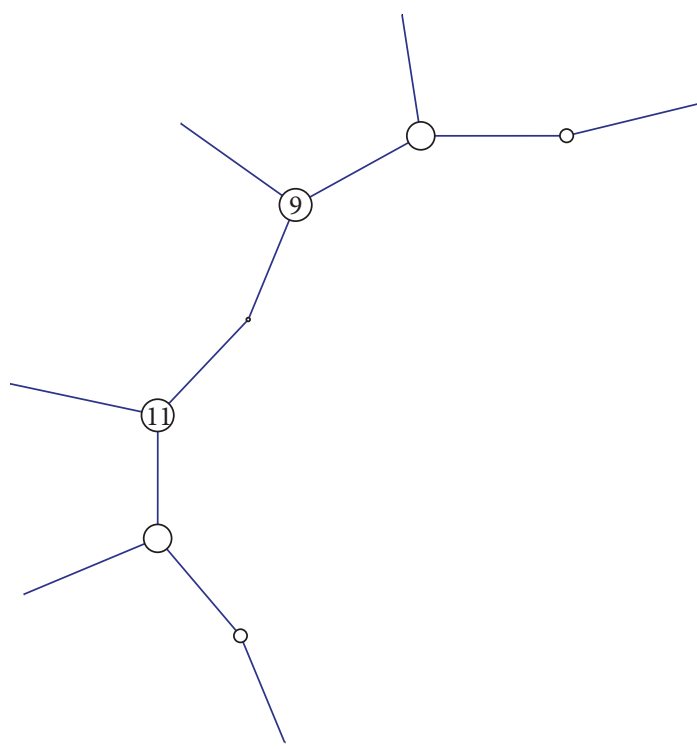

(a)

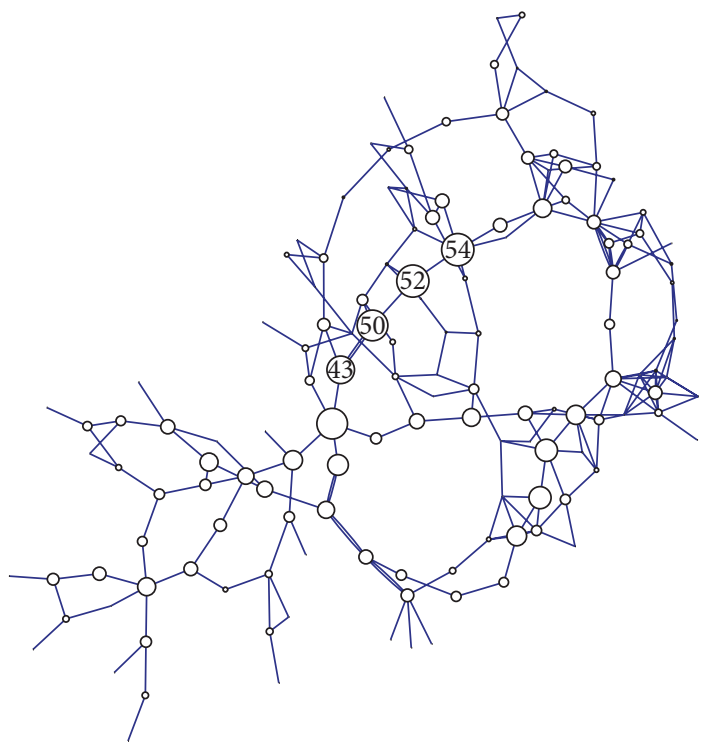

(c)

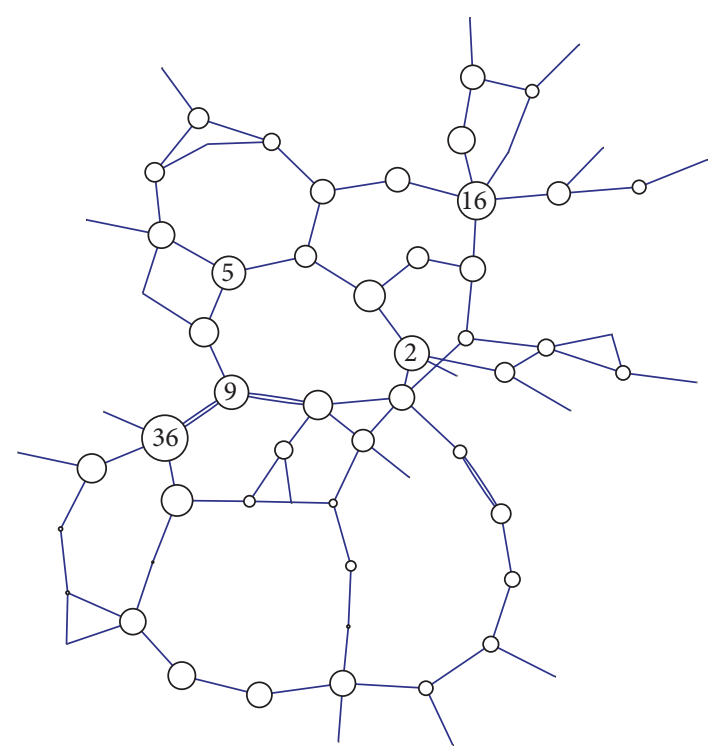

(b)

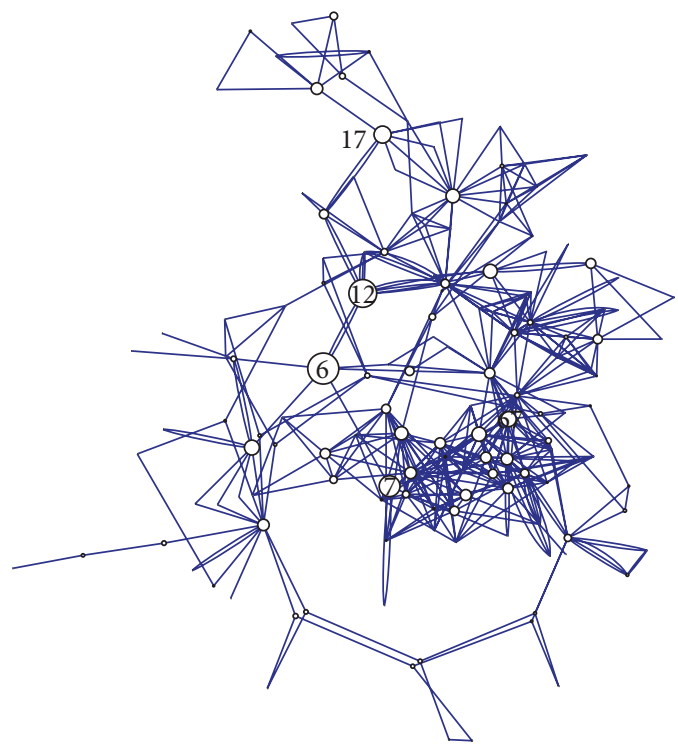

(d)

Figure 8: Multigraphs of the test power grids (a) $\mathscr{G}_{4}$, (b) $\mathscr{G}_{16}$, (c) $\mathscr{G}_{48}$, and (d) $\mathscr{G}_{50}$.

to the network it belongs to, and it is prohibitive to compare two different networks just using this metric. Because of this, global efficiency is used as a measure of vulnerability $[7,8]$ within a network, and a similar philosophy to evaluate the global efficiency when critical nodes are disconnected or attacked is used here.

To analyze the vulnerability in all test power grids, two scenarios are proposed.

(1) Targeted attacks. In this scenario, lines connecting nodes with higher centrality indices are disconnected until each node is completely isolated from the power grid. A list of these nodes is given in Table 3.

(2) Random attacks. Lines disconnected belong to nodes chosen randomly with a uniform probability distribution.
TABLE 3: Nodes with higher centrality in each test power grid.

\begin{tabular}{ccc}
\hline $\mathscr{G}$ & $C_{B}$ & $C_{C}$ \\
\hline 4 & 9,11 & 9,11 \\
16 & $36,16,2,9,5$ & $37,65,68,2,9$ \\
48 & $54,52,50,37,43$ & $86,130,132,52,50$ \\
50 & $6,12,7,17,67$ & $6,7,12,139,66$ \\
\hline
\end{tabular}

Figure 10 shows the results of both scenarios for each test power grid. Figure 10(a) illustrates that both closeness and betweennes based attacks result in the same drop in 


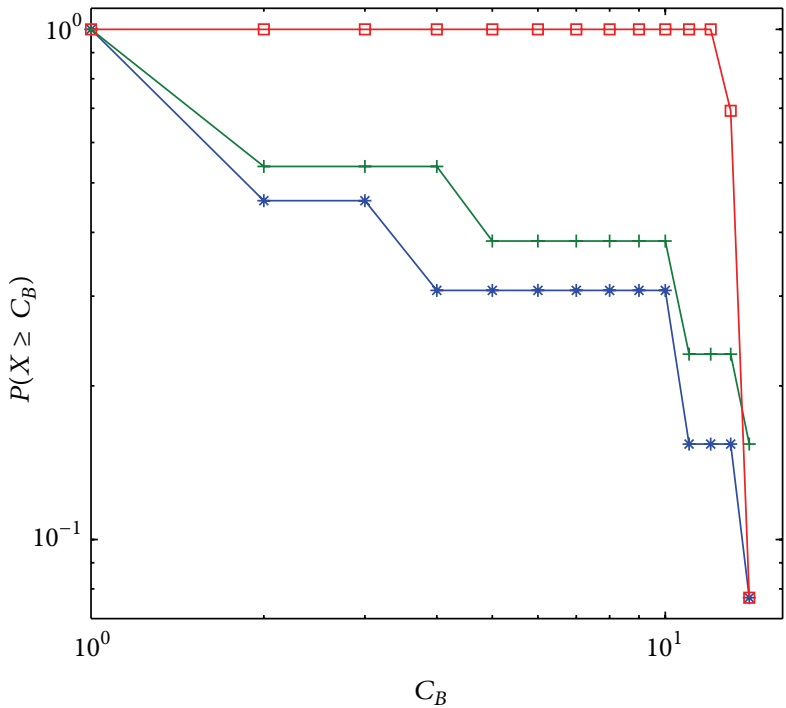

(a)

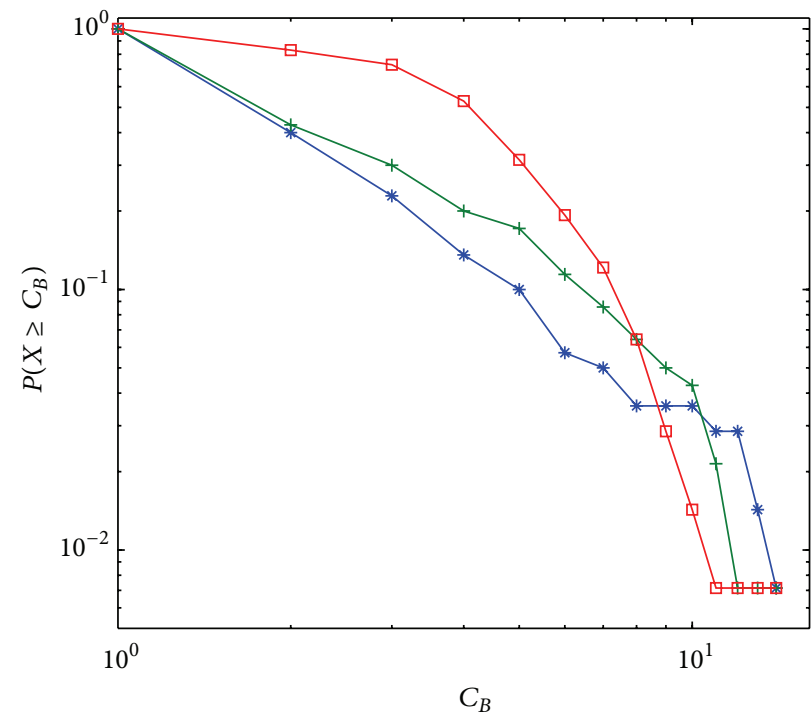

(c)

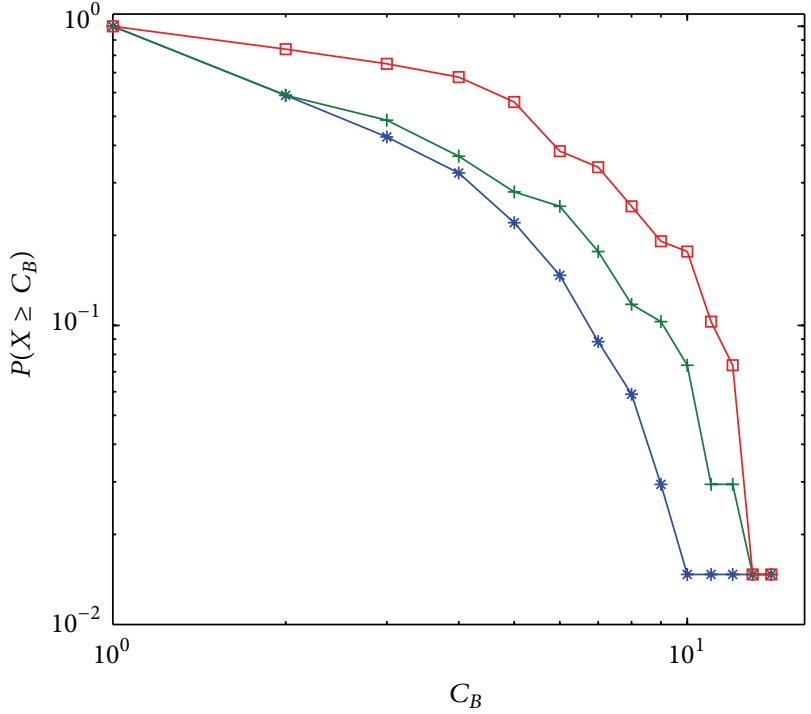

(b)

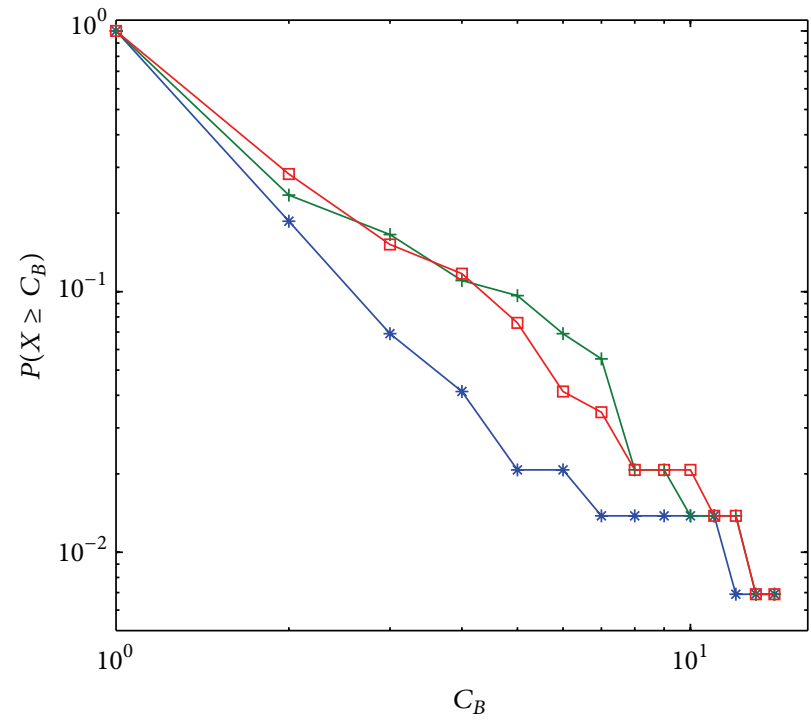

(d)

Figure 9: CDF of the betweenness index of (a) $\mathscr{G}_{4}$, (b) $\mathscr{G}_{16}$, (c) $\mathscr{G}_{48}$, and (d) $\mathscr{G}_{50}$ obtained using three different definitions: the original definition -+—, the proposed modification $-*$ - and the centrality based on $\left|z_{i i}\right|-\square-$.

global efficiency. This is due to the fact that nodes 9 and 11 are, at the same time, the closest ones and those with bigger betweenness, as can be seen in Table 3. A similar situation can be spotted in Figure 10(d), with nodes 6 and 12 .

Since global efficiency is a function of all shortest paths in a power grid, a node with high betweenness that affects a considerable portion of these paths ( $N$ times the number of shortest paths it lies on) produces a big decrease in global efficiency, while a low decrease results from a node with high closeness that usually influences a small number of paths (at least $N$ and at most $N$ times the number of shortest paths it lies on). In this way, the vulnerability analysis performed in this section, although it does not take into account the modification of power flow in the grid, shows that nodes detected via centrality measures are critical to their respective power grid.

\section{Conclusions}

In general, power grids are exponential rather than scalefree, as shown from the results in Table 2, which makes them barely vulnerable if only its connection patterns are taken into account. This information is fundamental if a cascade failure model is defined for power grids.

The use of global efficiency shows that the modified centrality measures used in this paper identify critical or dominant nodes in a power grid. This information can 


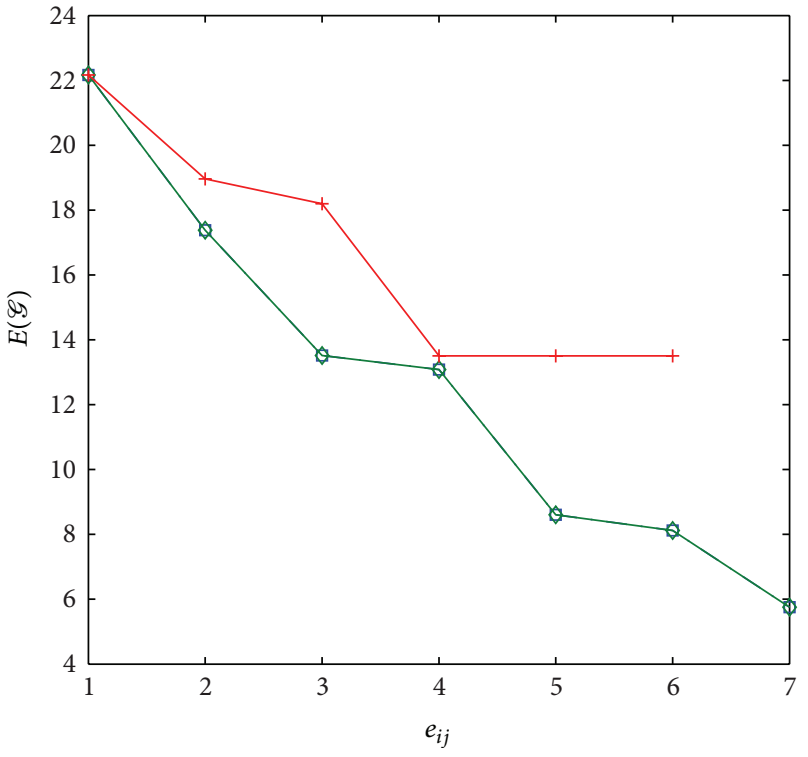

(a)

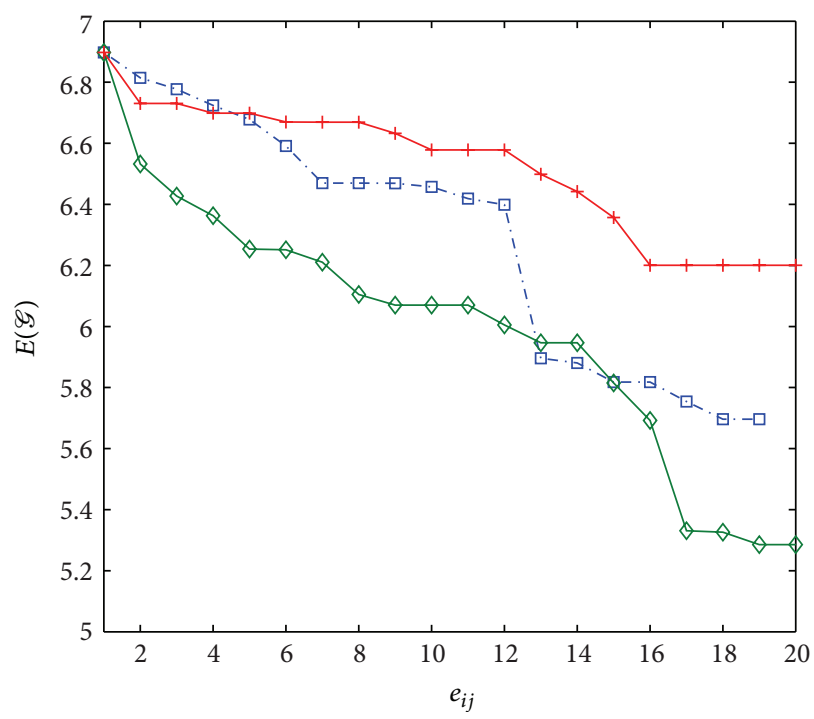

(c)

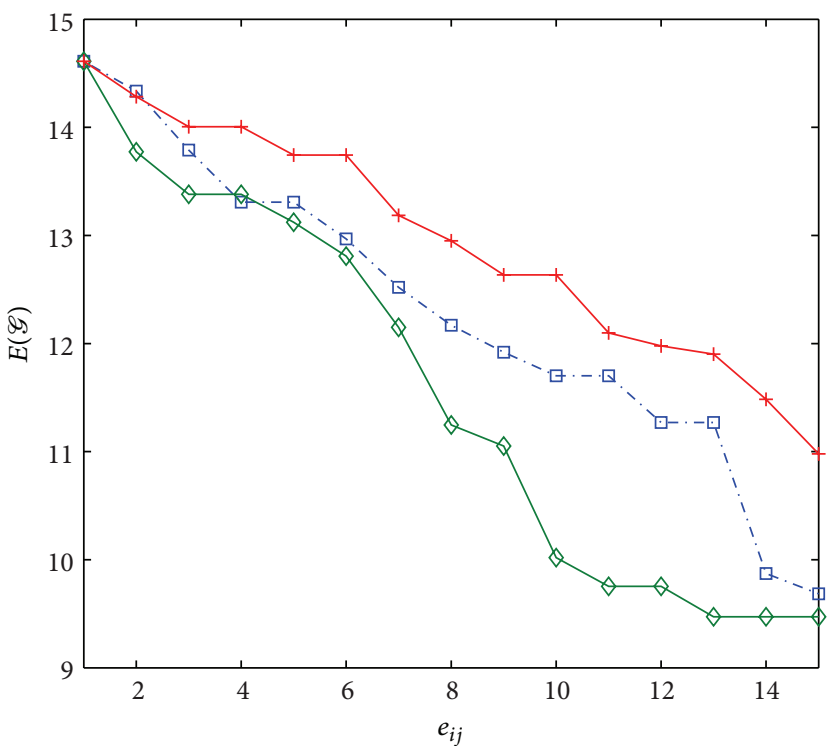

(b)

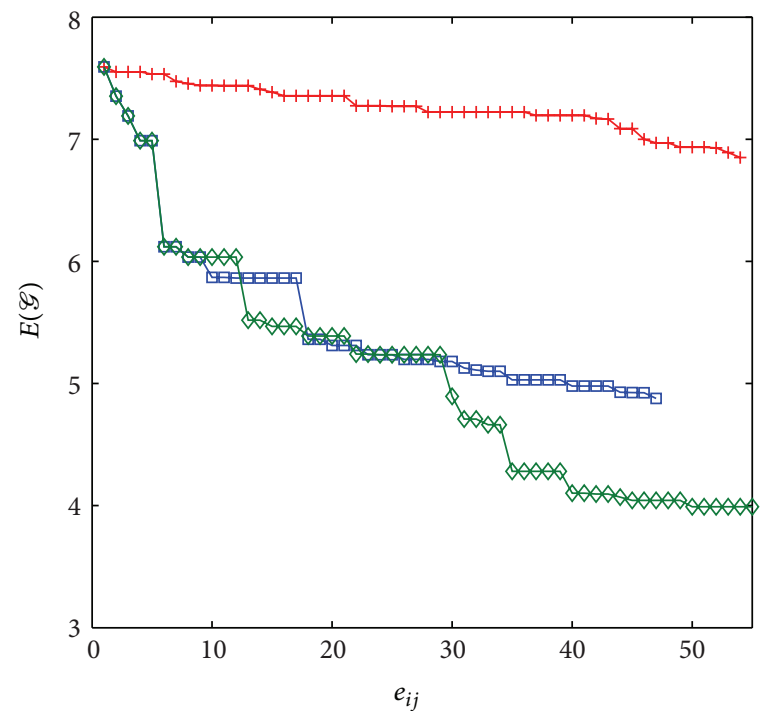

(d)

Figure 10: Global efficiency of (a) $\mathscr{G}_{4}$, (b) $\mathscr{G}_{16}$, (c) $\mathscr{G}_{48}$, and (d) $\mathscr{G}_{50}$ under random (-+-) and targeted attacks (-• $\square \cdot-$ closeness and — $\diamond$ betweenness based).

be used to filter credible contingencies before performing a contingency analysis or to define control allocation to regulate power transfer over specific corridors.

\section{References}

[1] California Energy Commission, "Smart grid cyber security potential threats, vulnerabilities and risks," PIER, Program Interim Project Report CEC-500-2012.047, 2012.

[2] Congress of United States Office of Technology Assessment, "Physical Vulnerability of Electric Systems to Natural Disasters and Sabotage," OTA-E-453, 1990.
[3] P. Gomes, "New strategies to improve bulk power system security: lessons learned from large blackouts," in Proceedings of the IEEE Power Engineering Society General Meeting, pp. 17031708, Denver, Colo, USA, June 2004.

[4] C. J. Kim and O. B. Obah, "Vulnerability assessment of power grid using graph topological indices," International Journal of Emerging Electric Power Systems, vol. 8, no. 6, article 4, pp. 1-15, 2007.

[5] V. Rosato, S. Bologna, and F. Tiriticco, “Topological properties of high-voltage electrical transmission networks," Electric Power Systems Research, vol. 77, no. 2, pp. 99-105, 2007.

[6] Y. Shao and J. Yu, "Priority evaluation of transmission lines based on vulnerability assemment," in Proceedings of the $1 s t$ 
International Conference on Sustainable Power Generation and Supply (SUPERGEN '09), pp. 1-6, April 2009.

[7] P. Crucitti, V. Latora, and M. Marchiori, "Locating critical lines in high-voltage electrical power grids," Fluctuation and Noise Letters, vol. 5, no. 2, pp. L201-L208, 2005.

[8] V. Latora and M. Marchiori, "Vulnerability and protection of infrastructure networks," Physical Review E, vol. 71, no. 1, Article ID 015103, 4 pages, 2005.

[9] S. Xu, H. Zhou, C. Li, and X. Yang, "Vulnerability assessment of power grid based on complex network theory," in Proceedings of the Asia-Pacific Power and Energy Engineering Conference (APPEEC '09), pp. 1-4, March 2009.

[10] G. Zhang, C. Wang, J. Zhang, J. Yang, Y. Zhang, and M. Duan, "Vulnerability assessment of bulk power grid based on complex network theory," in Proceedings of the 3rd International Conference on Deregulation and Restructuring and Power Technologies (DRPT '08), pp. 1554-1558, April 2008.

[11] E. Bompard, R. Napoli, and F. Xue, "Extended topological approach for the assessment of structural vulnerability in transmission networks," IET Generation, Transmission and Distribution, vol. 4, no. 6, Article ID IGTDAW000004000006000716000001, pp. 716-724, 2010.

[12] P. Hines and S. Blumsack, "A centrality measure for electrical networks," in Proceedings of the 41st Annual Hawaii International Conference on System Sciences (HICSS '08), p. 185, January 2008.

[13] D. Ban, "A flow-based centrality measure through resistance distances in smart-grid networks," in Proceedings of the 54th Annual IEEE Global Telecommunications Conference: "Energizing Global Communications" (GLOBECOM '11), Houston, Tex, USA, 2011.

[14] A. B. M. Nasiruzzaman, H. R. Pota, and F. R. Islam, "Complex network framework based dependency matrix of electric power grid," in Proceedings of the 21st Australasian Universities Power Engineering Conference (AUPEC '11), Brisbane, Australia, 2011.

[15] L. C. Freeman, "The gatekeeper, pair-dependency and structural centrality," Quality and Quantity, vol. 14, no. 4, pp. 585$592,1980$.

[16] G. E. Antonio, Análisis y Operación de Sistemas de Energía Eléctrica, McGraw-Hill, 2008.

[17] E. W. Dijkstra, "A note on two problems in connexion with graphs," Numerische Mathematik, vol. 1, no. 1, pp. 269-271, 1959.

[18] L. C. Freeman, "Centrality in social networks conceptual clarification,” Social Networks, vol. 1, no. 3, pp. 215-239, 1978.

[19] C. Correa, C. Tarik, K.-L. Ma, and K. Keeton, "The Derivatives of Centrality and their applications in Visualizing Social Networks," 2009.

[20] B. Toufik, "Modélisation de parcours du web et calcul de commnautés par émergence," Thèse Pour Obtenier le Diplôme de Doctorat Informatique, Université Montpellier II, 2005.

[21] R. Albert and A.-L. Barabási, "Statistical mechanics of complex networks," Reviews of Modern Physics, vol. 74, no. 1, pp. 47-97, 2002.

[22] L. C. Freeman, "A set of measures of centrality based on betweenness," Sociometry, vol. 40, no. 1, pp. 35-41, 1977.

[23] S. P. Borgatti and D. S. Halgin, "On network theory," Organization Science, vol. 22, no. 5, pp. 1168-1181.

[24] D. N. Kosterev, C. W. Taylor, and W. A. Mittelstadt, "Model validation for the august 10,1996 wscc system outage," IEEE Transactions on Power Systems, vol. 14, no. 3, pp. 967-979, 1999.
[25] A. Bavelas, "A mathematical model for group structure," Applied Anthropology, vol. 7, pp. 16-30, 1948.

[26] G. Sabidussi, “The centrality index of a graph," Psychometrika, vol. 31, no. 4, pp. 581-603, 1966.

[27] A. Fronczak, P. Fronczak, and J. A. Hołyst, "Average path length in random networks," Physical Review E, vol. 70, no. 5, Article ID 056110, 7 pages, 2004.

[28] D. J. Watts and S. H. Strogatz, "Collective dynamics of "smallworld" networks," Nature, vol. 393, no. 6, pp. 440-442.

[29] V. Larota and M. Marchiori, "Efficient behavior of small-world networks," Physical Review Letters, vol. 87, no. 19, Article ID 198701, 4 pages, 2001. 


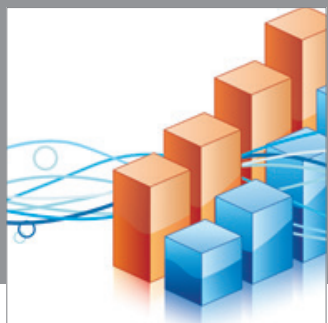

Advances in

Operations Research

mansans

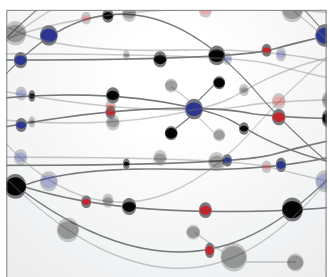

The Scientific World Journal
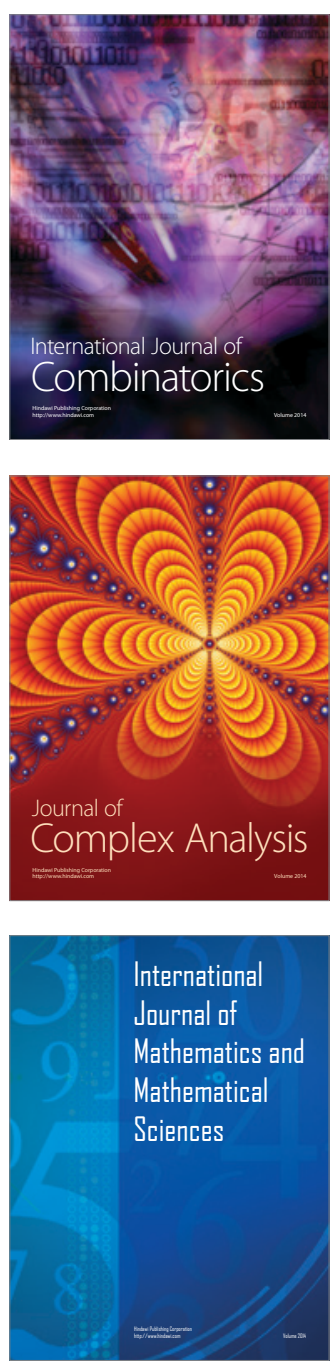
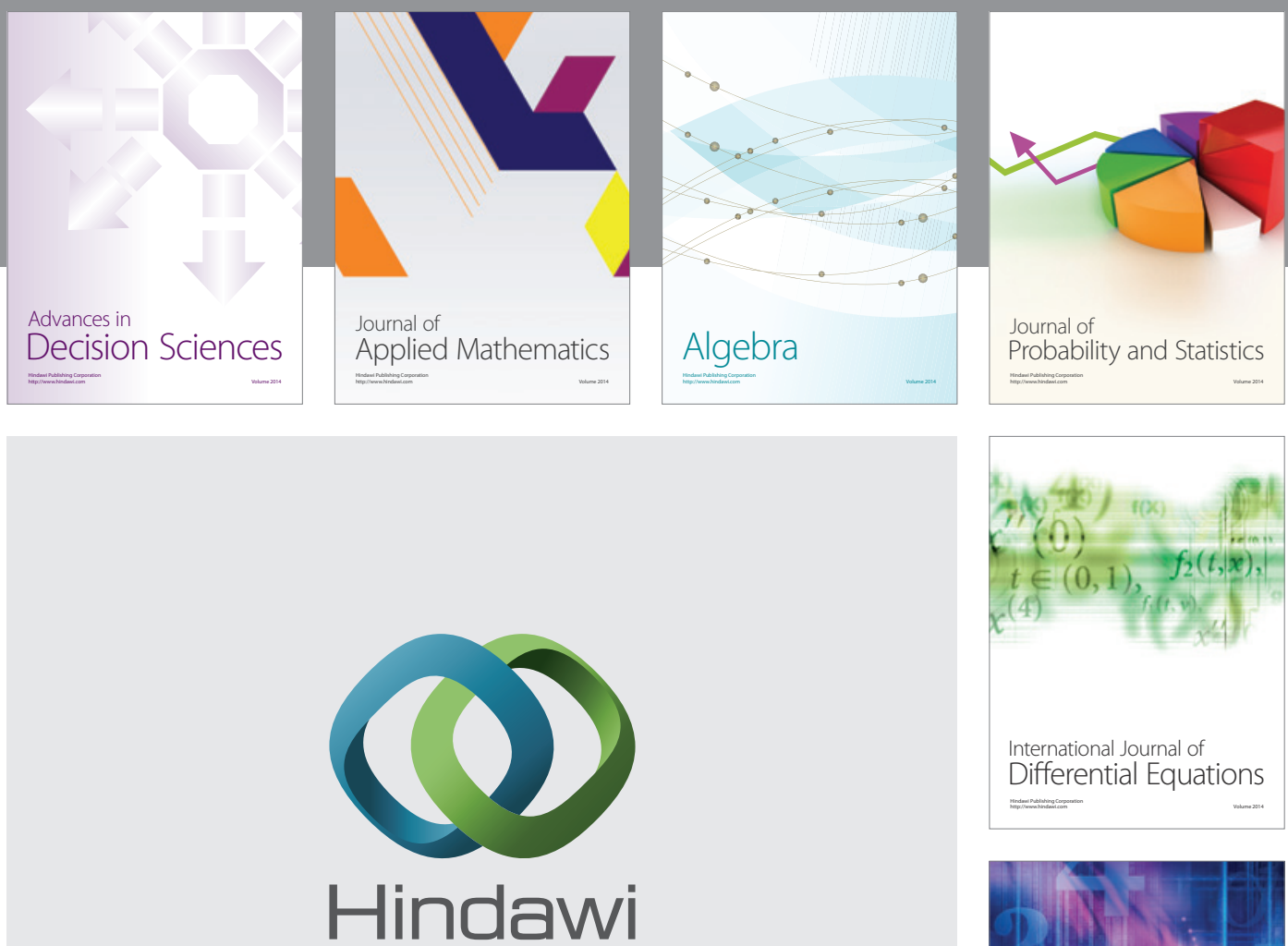

Submit your manuscripts at http://www.hindawi.com
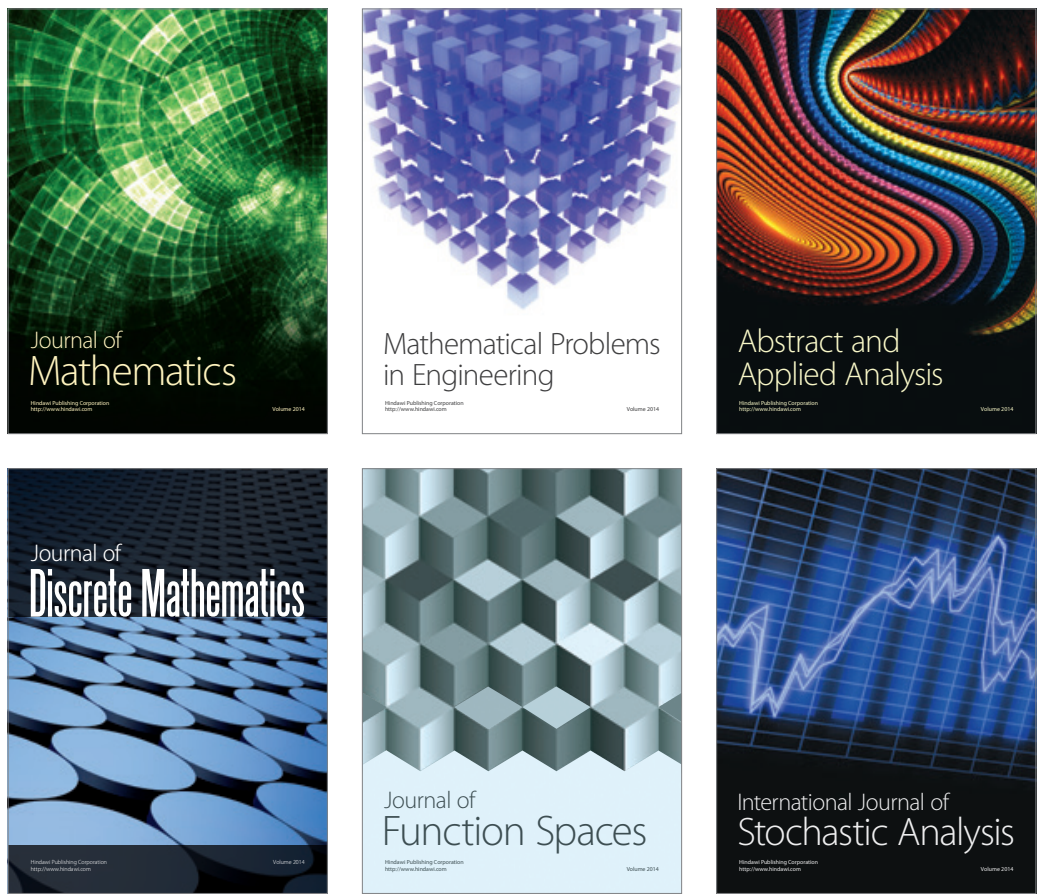

Journal of

Function Spaces

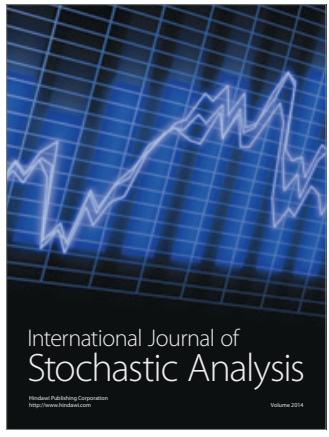

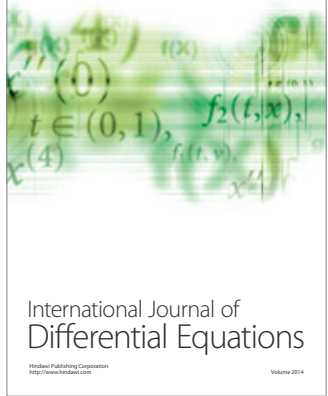
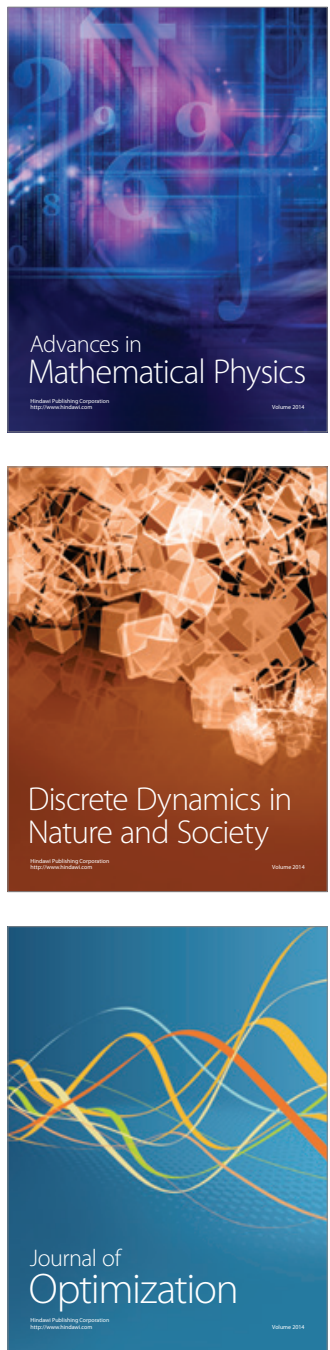